\title{
FAMILIARITY AND DISTRACTION AFFECT DRIVERS' RESPONSES TO IN-VEHICLE WARNINGS FOR A HAZARD THAT IS NOT YET VISIBLE
}

\author{
James W. Jenness, Amy K. Benedick, Richard W. Huey \\ Westat \\ Rockville, Maryland, USA \\ Email: jamesjenness@westat.com
}

\begin{abstract}
Summary: On-road experiments measured drivers' initial responses to in-vehicle emergency electronic brake light (EEBL) warnings indicating hard braking by some vehicle in the lane ahead. Participants drove within a platoon of four research vehicles on an Interstate highway with other traffic present. Speed and time gap between the participant's vehicle and the vehicle immediately ahead were measured before and after a warning was triggered. This paper examines the effects of prior training about EEBL warnings and driver distraction on responses to the warning when the hazard (braking vehicle in lane ahead) was blocked from view by an intervening vehicle. An EEBL warning, if effective, should encourage drivers to immediately reduce their speed and increase the time gap between their vehicle and the vehicle ahead. We hypothesized that the warning would be more effective for drivers who had received training about the meaning of EEBL warnings as compared to naïve drivers; and more effective for visually distracted drivers as compared to drivers who were looking ahead but saw no hazard when the warning was triggered. Results suggest that speed reductions following onset of the warning were increased by training and decreased by distraction. The EEBL warning elicited the most robust response from drivers who were not distracted and from those had been trained about the warning system. There was no evidence for an interaction between these two factors. These results suggest that training drivers about vehicle safety systems may increase proper behavioral responses, thereby increasing the effectiveness of in-vehicle warnings.
\end{abstract}

\section{INTRODUCTION}

Rear-end crash scenarios have been identified as a priority opportunity for development of vehicle-to-vehicle countermeasures (Najm, Toma, Brewer, 2013). Dedicated short range radio communications (DSRC) technology will make it possible for drivers to be warned about impending vehicle conflicts that are beyond the capabilities of collision avoidance systems that rely on conventional vehicle-based sensors. Emergency Electronic Brake Light (EEBL) is one of several proposed safety applications with high potential benefit that can be enabled through vehicle to vehicle communications (CAMP, 2005). An EEBL application will warn drivers about a vehicle braking hard in the lane ahead even if the braking vehicle's position is several vehicles ahead. This application is thought to be particularly useful when a driver's line of sight is obstructed by other vehicles or bad weather conditions.

EEBL may be viewed as a type of forward collision warning system (FCW). Normally, when a conventional FCW warning is triggered by vehicle-based sensors, a driver who looks immediately at the forward roadway will see the hazard (usually a stopped or slowing vehicle in close proximity ahead). When an EEBL warning is given, the alerted driver may not be able to 
see the other vehicle that is braking hard and triggering the warning. It is important to understand whether drivers react appropriately under these circumstances. Survey research has shown that vehicle owners often do not understand the capabilities and limitations of advanced vehicle systems (Jenness, et al., 2008; Braitman, et al., 2010; McDonald, et al., 2016), but there is a need to understand how drivers' knowledge (or lack of knowledge) about vehicle safety systems actually influences driving behavior.

The purpose of the present study was to examine drivers' initial responses to EEBL warnings in the first few seconds following onset of a warning for a hazard that was not visually verifiable. The EEBL warning, if effective, should encourage drivers to immediately start slowing down in response to the warning and increase the time gap between their vehicle and the vehicle immediately ahead. Two independent variables are considered in this paper: 1) Training whether or not the participant received training about EEBL warnings prior to the drive, and 2) Distraction - whether or not the participant was visually distracted by performing a manual radio tuning task at the moment when the EEBL warning was triggered. We hypothesized that drivers who had been trained about EEBL warnings would respond more robustly than drivers who had not been trained. We also hypothesized that distracted drivers would respond more robustly to the EEBL warning than the forward looking drivers. The rationale was that the forward looking drivers may feel that they have good situational awareness and may trust their own eyes more than the warning. They may judge the warning to be a false alarm and may not respond quickly. On the other hand, the distracted drivers may be aware that when they are performing a secondary task they have diminished situational awareness. Therefore, they may respond to the auditory component of the warning by slowing down, perhaps before they search the forward roadway for hazards. This would particularly be true for distracted drivers who had been trained about EEBL warnings. Understanding how drivers naturally respond to such warnings will enable researchers to develop design guidance for effective driver interfaces for such systems.

\section{METHOD}

\section{Design of Study}

This paper describes a two-factor (Training, Distraction) between subjects experiment that consists of a subset of experimental groups tested in a larger study. The four comparable treatment groups and number of participants in each group are:
1. Not Trained, Not Distracted $(\mathrm{n}=12)$
2. Not Trained, Distracted $\quad(\mathrm{n}=13)$
3. Trained, Not Distracted $\quad(n=13)$
4. Trained, Distracted $\quad(\mathrm{n}=14)$

On-road experiments were conducted on I-270 in Montgomery County, MD using a platoon of four research vehicles. Participants drove an instrumented vehicle (2011 Subaru Outback) and during the trip they were exposed to various in-vehicle driver assistance messages and EEBL warnings at predetermined locations along the roadway. Other vehicles in the platoon were driven by researchers (Figure 1). The first two vehicles in the platoon were used to stage simulated braking events and the third vehicle (Instrumented vehicle) was driven by the participant who was accompanied by a researcher riding in the back seat immediately behind the 
driver. A trailing vehicle, driven by another researcher, was used to maintain a safety buffer behind the participant. For safety reasons, braking events associated EEBL warnings were simulated. In-vehicle EEBL warnings were triggered simultaneously with automatic illumination of brake lamps on the Front vehicle in the platoon, but the Front vehicle did not actually brake during the warning events. As shown in Figure 1, a small vehicle (Nissan Sentra) positioned two vehicles ahead of the participant's Instrumented Vehicle "braked" but the brake lamps were not visible to the participant because the Intervening Vehicle immediately in front of the participant was large (Dodge Caravan) and obstructed the participant's view of the hazard.
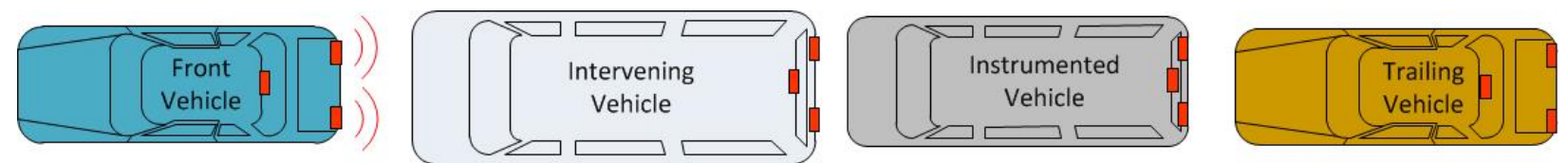

Figure 1. Configuration for an EEBL Event where hazard was not visually verifiable

\section{Participants}

Participants were recruited from the Washington, D.C. metropolitan area. Recruitment targeted drivers between the ages of 25-55. To be eligible for the study, potential participants had to have a valid driver's license from MD, VA, or DC, regularly drive a passenger vehicle at least 3-5 days per week, have 5 or more years of driving experience, and have a driving record that was in good standing. Participants were randomly assigned to treatment groups with constraints such that gender and age distributions would be similar across groups. Vehicle data from a few participants were missing or unusable. The final data set analyzed for the study included data from 52 participants. Approximately equal numbers of men and women participated in each study group and the mean age of male and female participants was each approximately 40 years. Participants were compensated $\$ 100$ for their time and travel expenses.

\section{Apparatus}

The study used an instrumented vehicle, a 2011 Subaru Outback fitted with driver and vehicle monitoring equipment including a Mobileye C2-270 system (Mobileye) which provided data on the instrumented vehicle's speed and time gap (SAE J2944) to the vehicle ahead. Researchers communicated wirelessly to stage all of the study events and the participant was not aware of upcoming events.

For each EEBL event, the participant received an audio and visual warning. The audio component of the EEBL warning was a tone presented as a triplet of four bursts lasting a total of 1.5 seconds. This alert had strong components in the $630 \mathrm{~Hz}$ and $5 \mathrm{kHz}$ third octave bands. It was played through the front stereo speakers at a consistent level (peak measured at $73.4 \mathrm{dBA}$ ). The visual component of the EEBL warning was a row of 36 red LED lights projected on the windshield to create a head-up display (HUD) (Figure 2, left). The lights remained on through each 5-second warning period. For non-warning events, the participant received an audio chime and a visual driver assistance message. The visual message was an icon and text displayed simultaneously on a monitor mounted at the top of the center stack. After 10 seconds of display time, the screen returned to a default view of menu items. Figure 2 (inset) shows one of the driver assistance messages presented to all participants. 


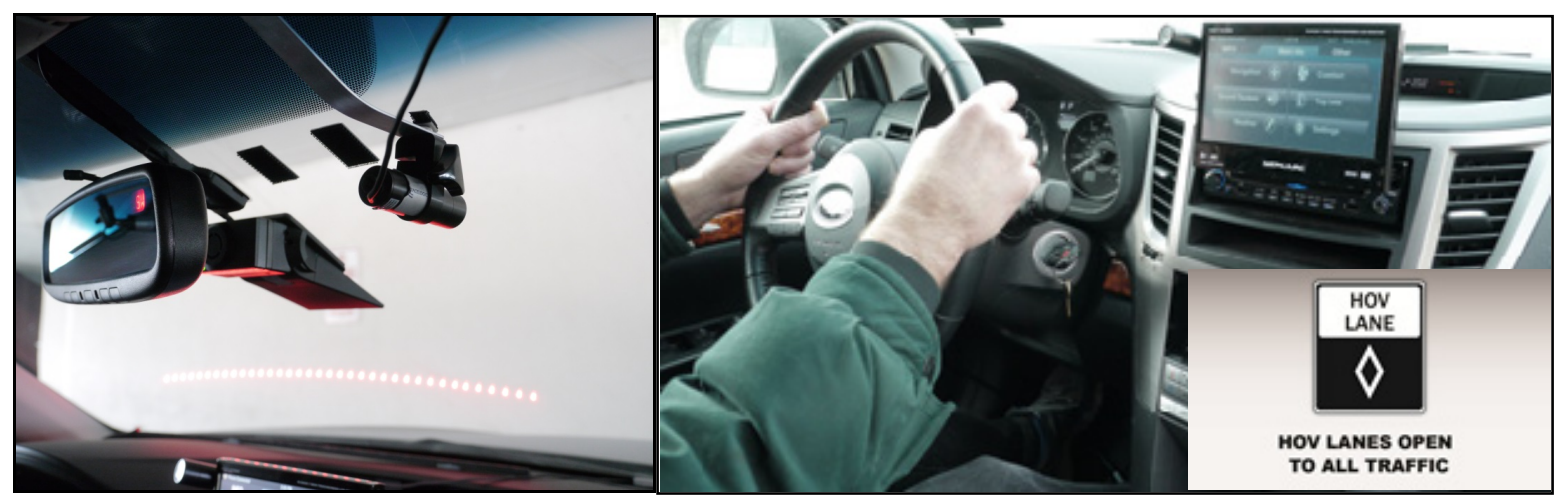

Figure 2. Visual component of EEBL warning reflected in windshield (left), view of default display screen on center stack (right) and example of driver assistance message (inset)

\section{Procedure}

Driving data were collected on Interstate I-270 between Exit 6 and Exit 22 in Montgomery County, MD between 10 am and 2 pm when traffic was free flowing and the weather was clear. Participants were told that they were conducting a test drive of a car that has a number of advanced features and that their task was to drive the vehicle to evaluate it and then discuss it after the drive. The participants were also told that the vehicle had some prototype safety features, including advanced warning systems for potential lane departures and forward collisions. Participants were told that during the drive their most important responsibility was to drive safely.

Participants in the trained groups received specific training about the EEBL warning system prior to the drive. This training consisted of watching a short video segment which explained the EEBL application (CAMP and U.S. Department of Transportation, 2011) and, prior to the test drive, the researcher triggered the EEBL warning so that the participant was familiar with the implementation of EEBL warnings in the test vehicle.

Prior to their test drive, participants in the distracted groups were asked to practice manually tuning a radio that had been installed in the center stack of the instrumented vehicle immediately above the climate control console and within easy reach of the driver. The radio had two knobs labeled "Volume" and "Tuning" and a digital display of the station frequency (e.g. 101.3). The radio was not connected to speakers so that the participants had to rely entirely on the visual display to complete the radio tuning task. In the practice session and five times during the test drive, a prerecorded voice instructed the participant to tune the radio to a new station: "Please tune the radio to 105.7" (for example). During the third radio tuning task, while the participant was looking at the radio, an EEBL warning was triggered.

A researcher accompanied the participant on the test drive. At a designated point during the drive, this researcher activated EEBL warnings in the Instrumented Vehicle. This warning was synchronized with computer-controlled activation of the brake lamps on the Front Vehicle (Figure 1). Other driver assistance messages were triggered automatically by GPS location and 
heading at designated points throughout the drive. These messages were designed to be believable events to help encourage the participant to view the system as real and reliable. Generally, participants maintained their speed during presentation of the driver assistance messages.

\section{RESULTS}

Each participant's speed data and time gap data were reduced by calculating a mean within each one-second bin and then normalizing the data to zero at the one-second interval immediately before onset of the EEBL warning. Figure 3 shows the mean (+/- 1 SEM) speed changes for each treatment group after onset of the first EEBL warning experienced during the drive. Figure 4 shows the mean time gap changes per group. The two figures show that all of the groups mean responses to the warning take several seconds to develop. Due to the normalization of all data at -.5 seconds, differences between the groups for speed changes and time gap changes are not apparent for the first few seconds after the warning was issued. The decrease in speed is greatest approximately 5-7 seconds after onset of the warning.

A repeated measures AVOVA (SAS 9.4 for Windows; Proc GLM) was conducted to assess the speed change data across time bins centered at 3.5, 4.5, 5.5, and 6.5 seconds. Training and Distraction were included as between Ss factors. After determining that there was no evidence for a Training-by-Distraction interaction, $\mathrm{F}(1,48)=.01, \mathrm{p}=.92$, this factor was dropped from the model. There were significant main effects of Distraction, $F(1,49)=4.78, p=.03$ and Training, $\mathrm{F}(1,49)=4.86, \mathrm{p}=.03$, and Time $\mathrm{F}(3,147)=5.61, \mathrm{p}=.001$. Interactions (Time-by-Distraction) and (Time-by-Training) were not statistically significant over the period examined.

A similar repeated measures AVOVA was conducted to assess the time gap changes. After determining that there was no evidence for a Training-by-Distraction interaction, $\mathrm{F}(1,48)=.02, \mathrm{p}$ $=.89$, this factor was dropped from the model. There was no significant effect of Distraction, $\mathrm{F}(1,49)=1.98, \mathrm{p}=.17$; a significant effect of Training, $\mathrm{F}(1,49)=11.8, \mathrm{p}=.001$, and a significant effect of Time $\mathrm{F}(3,147)=21.3, \mathrm{p}=.0001$. There was a significant Time-byDistraction interaction $\mathrm{F}(3,147)=3.53, \mathrm{p}=.02$ and a significant Time-by-Training interaction $\mathrm{F}(3,147)=7.8, \mathrm{p}=.0001$.

As can be seen in Figure 3, the groups that had been trained about EEBL slowed down more than groups that were not trained. Also, participants who were looking forward when the warning was triggered slowed down more than participants who were distracted by the radio tuning task. The group that slowed the least was the Not Trained / Distracted group and the group that slowed down the most was the Trained / Not Distracted group.

A similar ordering of groups can be seen with time gap changes (Figure 4). The Trained / Not Distracted group increased their time gap the most and the Not Trained / Distracted group exhibited the smallest increase in time gap. 


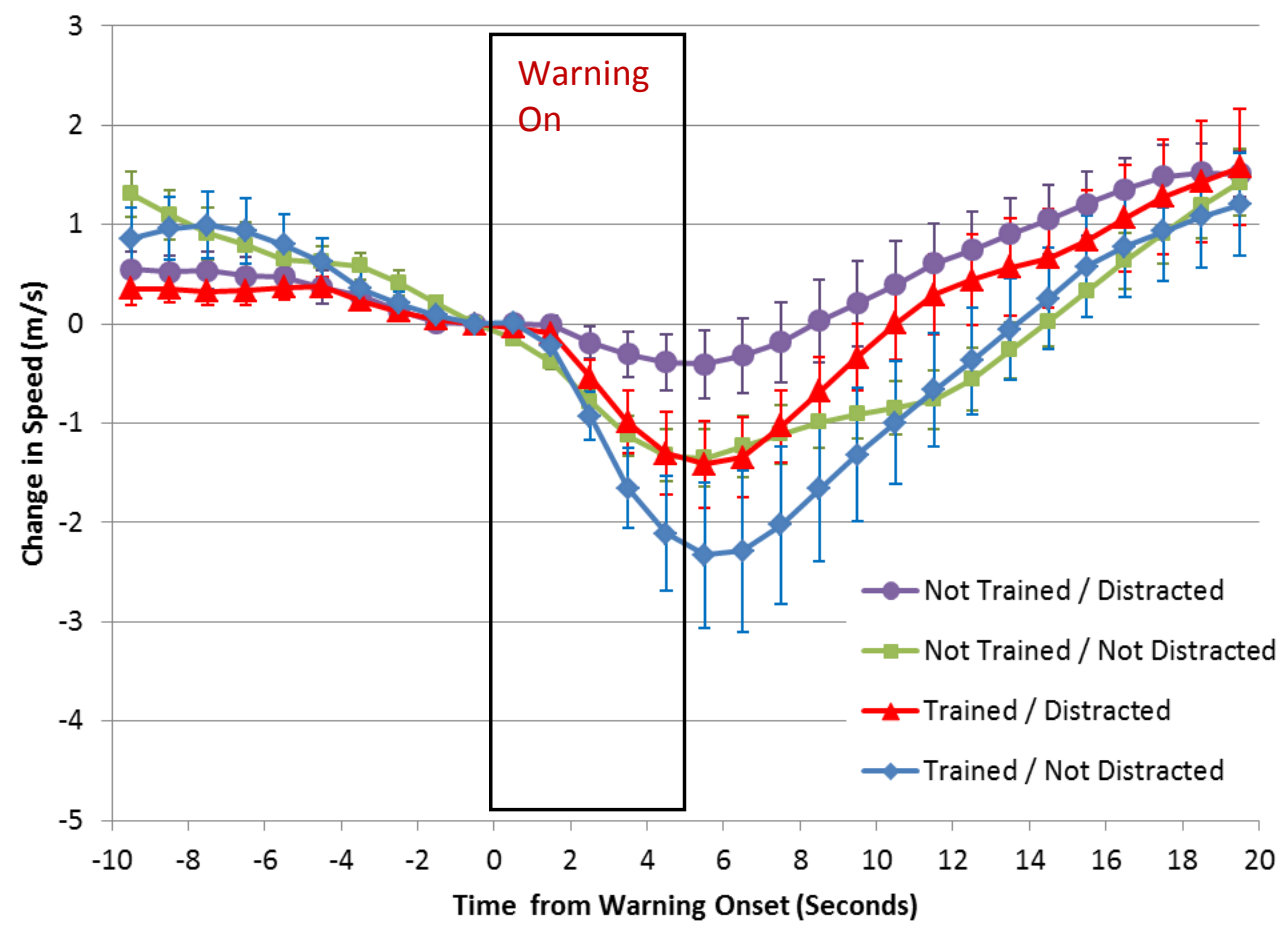

Figure 3. Mean speed changes following EEBL warning

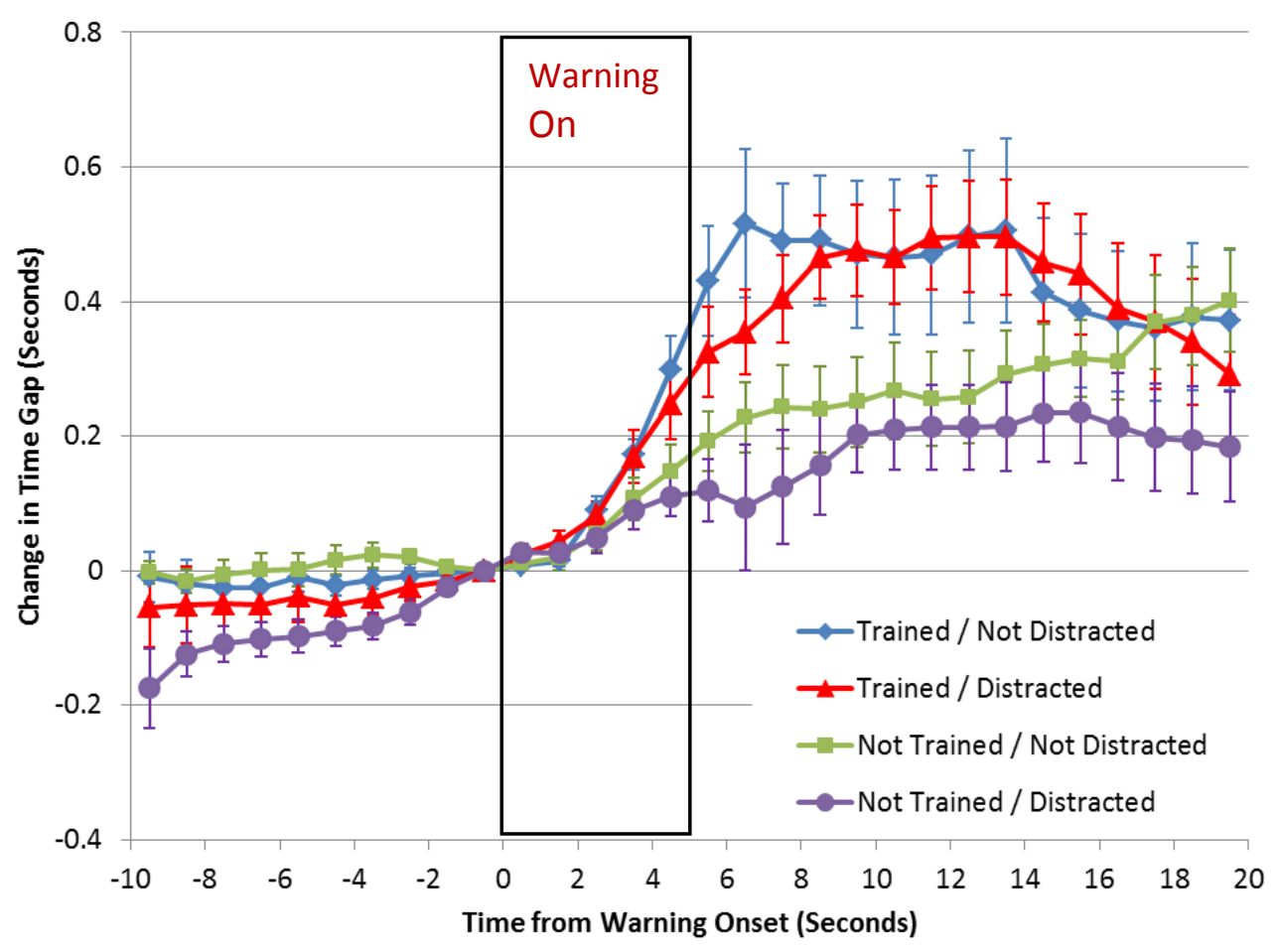

Figure 4. Mean time gap changes following EEBL warning 


\section{DISCUSSION}

Unlike conventional FCWs, EEBL warnings, as well as other proposed vehicle-to-vehicle collision warnings may be triggered when there is no immediate visual confirmation of the threat, but the driver needs to respond quickly and appropriately within a few critical seconds after onset of the warning. The present study developed a safe, on-road protocol for assessing initial driver behaviors with respect to in-vehicle EEBL warnings.

The hypothesis that familiarity with EEBL warnings (training) would lead to more robust slowing responses was confirmed by the data. However, we expected that distracted participants would show more vigorous responses or at least equally vigorous responses as participants who were already looking forward. In fact, the data did not support this hypothesis, which is a concern from a safety perspective. Subjective data, not presented here, indicated that a few distracted participants initially attributed the EEBL warning sound to something that they caused by touching the radio controls. This may indicate a methodological limitation of the radio tuning task as it was implemented in his study.

\section{ACKNOWLEDGMENTS}

This research was funded by National Highway Traffic Safety Administration under contract DTNH22-11-D-00237/007 (Christian Jerome, COTR). The views expressed in this work are those of the authors and do not represent official NHTSA policy.

\section{REFERENCES}

Braitman, K.A., A.T. McCartt, D.S. Zuby, and J. Singer. (2010).Volvo and Infiniti Drivers' Experiences with Select Crash Avoidance Technologies. Traffic Injury Prevention, 11, 270 278.

CAMP \& U.S. Department of Transportation (2011). Vehicle-to-Vehicle Communication. The Future of Vehicle Crash Avoidance [DVD]. Washington, DC: U.S. Department of Transportation.

CAMP (2005). Vehicle Safety Communications Project Task 3 Final Report. Identify Intelligent Vehicle Safety Applications Enabled by DSRC (Report No. DOT HS 809 859). Washington, DC: U.S. Department of Transportation, National Highway Traffic Safety Administration.

Jenness, J.W., N.D. Lerner, S. Mazor, J.S. Osberg, and B.C. Tefft. (2008). Use of Advanced InVehicle Technologies by Younger and Older Early Adopters: Selected Results for Five Technology Surveys. DOT-HS-811-004. Washington, DC: U.S. Department of Transportation.

McDonald, A.B., D.V. McGehee, S.T. Chrysler, N.M. Askelson, L.S. Angell, and B.D. Seppelt (2016). National Survey Identifying Gaps in Consumer Knowledge of Advanced Vehicle Safety Systems. Transportation Research Record, 2559, 1-6.

Najm, W.G., Toma, S., and Brewer, J. (2013). Depiction of Priority Light-Vehicle Pre-Crash Scenarios for Safety Applications Based on Vehicle-To-Vehicle Communications. (Report No. DOT HS 811 732). Washington, DC: U.S. Department of Transportation, National Highway Traffic Safety Administration. 